\title{
Prevalence of Macroprolactin in Hyperprolactinemic Patients Receiving Anti-Psychotics
}

\author{
Loai Ali Al Mortada Al Wasify ${ }^{1 *}$, Shaikha Said Al Maamary ${ }^{1}$ and Mohammed Nasser Rashid Al \\ Tobi $^{2}$ \\ ${ }^{1}$ Al Masarra hospital, Laboratory Department, $\mathrm{MOH}$, Sultanate of Oman \\ ${ }^{2}$ Sultan Qaboos University, College of Medicine, Laboratory Department, Sultanate of Oman \\ *Corresponding author: Loai Ali Al Mortada Al Wasify, Al Masarra hospital, Laboratory Department, MOH, Sultanate of Oman
}

ARTICLE INFO $\quad$ ABSTRACT

Received: 慧 July 14, 2021

Published: 幽 July 27, 2021

Citation: Loai Ali Al Mortada Al Wasify, Shaikha Said Al Maamary, Mohammed Nasser Rashid Al Tobi. Prevalence of Macroprolactin in Hyperprolactinemic Patients Receiving Anti-Psychotics. Biomed J Sci \& Tech Res 37(3)-2021. BJSTR. MS.ID.006015.

Abbreviations: PRL: Prolactin; MaPRL: Macroprolactin; GFC: Gel Filtration Chromatography; PEG: Polyethylene Glycol; CV: Coefficient of Variation
Background: Prolactin (PRL) hormone circulates in the blood in three forms, mono-prolactin which constitutes $85 \%$ of prolactin in healthy and hyperprolactinemia conditions, dimeric prolactin (big PRL), and polymeric PRL (big-big prolactin or Macroprolactin). Macroprolactin in normal conditions is not exceeding $2 \%$ of the total serum prolactin and had no biological activity. In some cases, of hyperprolactinemia, the dominant form becomes MaPRL and exceeding the $2 \%$ percentage of total leading to misdiagnosis of hyperprolactinemia and unnecessary radiological investigations. This study aims to detect the prevalence of MaPRL in Psychiatric patients with hyperprolactinemia due to antipsychotic medications at Al Masarra hospital.

Material and Methods: The study was conducted on 190 samples from patients with high prolactin in Al Masarra Hospital either inpatients or out-patient clinics either male or non-lactating, not pregnant female. The measurement of the Prolactin level was measured by the automated analyzer COBAS e411, Roche Diagnostic. Macroprolactin was precipitated by using Polyethylene glycol (PEG).

Results: Prevalence of Macroprolactin was $10.5 \%$ of hyperprolactinemic patients receiving antipsychotics. There was a statistically significant difference in gender between the symptomatic and asymptomatic groups. There was no significant difference in medications used between the symptomatic and asymptomatic group and there was a statistically significant difference in total Prolactin \& Macroprolactin between males and females.

Conclusion: Investigation for Macroprolactin should be done in every hyperprolactinemia patient who is receiving antipsychotics more especially the asymptomatic cases to avoid unnecessary radiological imaging and treatment.

\section{Introduction}

Prolactin hormone is a globular protein, synthesized and secreted by lactotrophs in the anterior pituitary gland [1]. The main functions of prolactin are exerted during pregnancy, enabling breast development, milk production \& lactation. Prolactin (PRL) has other biological functions as osmoregulation and immunoregulation [2]. PRL facilitates the maturation of T cells via IL-2 receptor expression, impairs B cell tolerance to self-antigens through the anti-apoptotic effect, develops antigen-presenting cells, and enhances immunoglobulin production [3]. Prolactin circulates in the blood in different forms; monomeric PRL with molecular weight $23 \mathrm{kDa}$ (referred to as little PRL), and it is the immunologically and biologically active form, dimeric PRL with molecular weight 48 to $56 \mathrm{kDa}$ (big PRL) which is biologically inactive, and polymeric form of PRL with a molecular weight $>100$ kDa (big-big PRL) which is also called a macroprolactin (MaPRL) [4]. The predominant isoform of PRL in healthy people, and also 
in most individuals with hyperprolactinemia, is a monomeric molecule that amounts to more than $85 \%$ of circulating hormone. However, in some patients with hyperprolactinemia, the dominant form becomes MaPRL, which in normal conditions does not exceed $2 \%$ of total serum PRL [5].

The increase in serum Prolactin concentration (hyperprolactinemia) is caused physiologically by pregnancy, lactation, stress \& pain and pathologically by a pituitary adenoma (secreting PRL), hypothyroidism, chest wall disease, hepatorenal disorders, and drug-induced (anti-dopaminergic drugs). The most common drugs that cause hyperprolactinemia are the antipsychotic medicines like risperidone, haloperidol, and olanzapine [6]. However, 29\% of hyperprolactinemia has been classified as idiopathic, because the causes are unknown [7] Anti-PRL autoantibody was found to be one of the major causes of idiopathic hyperprolactinemia [8]. In most cases, MaPRL is composed of immune complexes of PRL and anti-PRL autoantibodies, and $87 \%$ of MaPRL was PRL-IgG complex and $67 \%$ of MaPRL was autoantibody-bound PRL. Anti-PRL autoantibodybound PRL is a major form of PRL-IgG complex and PRL - IgG complex is a major form of macroprolactin [9]. It has been shown that MaPRL is biologically inactive because the large molecular size of this complex prevents its crossing through the capillary blood barrier and reaching target cells and immunoglobulin connecting with a specific epitope of PRL molecule may reduce the binding of the hormone to its receptors $[10,11]$. So, MaPRL has longer renal clearance resulting in its accumulation in the serum. In addition, the most available immunoassays used to measure PRL level do not distinguish MaPRL from monomeric PRL, which can lead to an incorrect diagnosis of hyperprolactinemia and unnecessary imaging study and treatment.

If MaPRL has been found to predominate in human serum, it will be called macroprolactinemia. In many studies, it is found in $10-45 \%$ of hyperprolactinemic patients, depending on the immunoassay used in the laboratory [12-15]. Although most of the studies reported that MaPRL is inactive, some studies reported that patients with a high concentration of MaPRL exhibit signs or symptoms of hyperprolactinemia such as galactorrhea, menstrual irregularities, or infertility, other studies reported that macroprolactinemia cannot be differentiated from hyper PRL based on clinical symptoms alone, because several of the signs and symptoms of hyperprolactinemia are non-specific, the occurrence of symptoms with macroprolactinemia may be coincidental [16-18]. It is important to identify the presence of MaPRL as the cause of hyperprolactinemia to avoid unnecessary radiological investigations and treatment with dopamine agonist medicines. Macroprolactin is found to interfere with most commercially available immunoassays used for prolactin. As a result, false high prolactin values (apparent hyperprolactinemia) are obtained, and these values depend on the assay method employed. So, the most available immunoassays used to measure PRL level do not distinguish MaPRL from monomeric PRL, which can lead to an incorrect diagnosis of hyperprolactinemia and unnecessary imaging study and treatment [19].

For detection of MaPRL, Gel filtration chromatography (GFC) is the gold standard method for quantification of the three variants of PRL, but this method is costly, time-consuming, and laborintensive, so it is not used in routine screening for MaPRL $[14,20]$. Laboratories generally use polyethylene glycol (PEG) precipitation to differentiate macroprolactinemia from true hyperprolactinemia. This method is simple and inexpensive and has been extensively validated against GFC. This study aims to evaluate the prevalence of MaPRL in hyperprolactinemic patients in Al Masarra hospital who are receiving antipsychotic medications by precipitating MaPRL with PEG.

\section{Materials \& Methods}

\section{Patients}

The study was done on 190 samples from patients with high prolactin levels and was receiving antipsychotics either male or non-pregnant non-lactating female from outpatient clinics or in-patients' wards of the Al Masarra hospital which is a tertiary psychiatric hospital in Sultanate of Oman from March 2020 to August 2020.

\section{Immunoassay}

The measurement of the Prolactin level was measured by the automated analyzer COBAS e411, Roche Diagnostic. By using the electrochemiluminescence immunoassay "ECLIA" based on the sandwich principle, using biotinylated monoclonal specific antibody interacted with streptavidin-coated microparticles and second antibody which is monoclonal antibody labeled with a ruthenium complex. After the application of voltage, the chemiluminescent emissions were measured. The samples were separated and stored at $-70 \mathrm{C}^{0}$ till processing.

The reference range was: $102-496 \mu \mathrm{IU} / \mathrm{mL}$ for non-pregnant women and $86-324 \mu \mathrm{IU} / \mathrm{mL}$ for men. The inter-assay coefficient of variation $(\mathrm{CV})$ was $2.2 \%$ and the intra-assay $\mathrm{CV}$ was $2.5 \%$.

\section{PEG-Precipitation}

Preparation of $25 \%$ solution of PEG 6000 was prepared by dissolving 25 grams of PEG 6000 in $60 \mathrm{ml}$ of distilled water at room temperature and mixing with vortex, then fulfilling the volumes till $100 \mathrm{ml}$ of solution. The prepared solution is stable for three months maximum period at $4 \mathrm{C}^{0}$. In a separate glass tube, an equal amount of serum sample and $25 \%$ of PEG were added. After thorough vortex mixing for a minute and stabilization for 30 minutes, the solution 
was centrifuged at $9500 \mathrm{xg}$ for 10 minutes. The supernatant portion of the sample was separated and prolactin level was measured in it. The prolactin level in the treated sample was multiplied by two to correct for the dilution with PEG. We followed the same protocol of Ana Maia Silva et al; 2014 in preparation of PEG solution and samples [21].

\section{Measuring Free-PRL}

The free prolactin is the prolactin level which will be recovered after precipitation of MaPRL by PEG.

The macroprolactin level will be determined using the following formula:

$$
\text { Macroprolactin } \%=\frac{(\text { SERUM PRL }- \text { PRL }(\text { SUPERNATANT }))}{\text { SERUM PRL }} \times 100
$$

\section{Interpretation}

- Free prolactin recovering less than $40 \%=$ significant presence of MaPRL.

- $\quad$ Free PRL recovering that will exceed 50\% = Monomeric PRL predominance.

- Free PRL recovering between $40 \%-50 \%=$ intermediate or at the grey zone [22-24].

\section{Statistical Analysis}

All data were recorded and entered in a statistical package on a compatible computer and varied. Analysis was done using an SPSS$20^{\text {th }}$ version. The results were tabulated, grouped and statistically analysed using the following tests:

1. Descriptive statistics (for quantitative data)

- $\quad$ Mean $\left(\mathrm{X}^{-}\right)$and standard deviation $( \pm \mathrm{SD})$

- $\quad$ Frequency with percentage (for qualitative data)

2. Student Test: A statistical test used to test for a significant of an independent variable in experiments where there are only two levels of this variable (to compare between two independent means).

3. Chi-square $\left(\mathbf{X}^{2}\right)$ : Was used to test the significant of the difference between the frequencies of the different observations i.e. qualitative data.

4. Spearman Correlation Test (r): was used when studying the relationship (direction and power) of quantitative variables simultaneously.

5. Logistic Regression Analysis: used to examine the extent to which a set of variables independently predicts a dependent variable.
6. $\boldsymbol{P}$ value: Used to indicate the level of significance:

- $\quad P>0.05$ : Insignificant

- $\quad P<0.05$ : Significant

- $\quad P<0.01$ : Highly significant

- $\quad P<0.001$ : Very highly significant

\section{A. Descriptive Statistics}

1. Quantitative Data: mean and standard deviation "SD" were used to measure central tendency and dispersion.

2. Qualitative Data: frequency of occurrence was calculated by number $(\mathrm{N})$ and percentage $\%$.

\section{B. Analytical Statistics}

1. Comparing between groups was done using:

$>$ Pearson Chi square- test $\left(\mathrm{X}^{2}\right)$ : for qualitative data.

$>$ Student's T test for quantitative data of two independent samples

2. Logistic regression analysis was done.

3. The level of significance was taken at $(0.05)$. So, $p$ value $>0.05$ was insignificant and $p$ value $\leq 0.05$ was significant.

4. The results were presented in tables and figures.

\section{Results}

The study showed that of 190 patients the mean age was $33.2 \pm 9.2$ years with the age range $16-62$ years. There were 149 females (78.4\%) and 41 males (21.6\%). 169 (88.9\%) were Omani and 21 (11.1\%) were non-Omani. The study found that $75.8 \%$ of hyperprolactinemia patients were asymptomatic and $24.2 \%$ were symptomatic. Meanwhile, (56.6\%) of patients received risperidone, (29.3\%) of patients received haloperidol, $(9.4 \%)$ of patients received flupentixol decanoate and (4.7\%) of patients received olanzapine. We found that (10.5\%) of hyperprolactinemia patient had macroprolactin predominance with recovery rate (RR) was between $25.6 \%-39.8 \%$ and $(3.2 \%)$ of cases were borderline with RR was between $44.3 \%$ - 50\%, while (86.3\%) of cases had free PRL with RR was between $55 \%$ - 98.9\%. Meanwhile, 25\% of MaPRL cases were males and $75 \%$ of the case were females. There was a statistically significant difference in gender between the symptomatic and asymptomatic group as males represented (28.5\%) of the studied sample in the asymptomatic group and $(0.0 \%)$ in the symptomatic group while females were $(71.5 \%)$ in the asymptomatic group in comparison to $(100.0 \%)$ in symptomatic group $(\mathrm{p}=0.00)$. There was a statistically significant difference in nationality between the symptomatic and asymptomatic group as Omani patients represented $(92.4 \%)$ of the studied sample in 
the asymptomatic group and (78.3\%) in the symptomatic group while non-Omani were less (7.6\%) in the asymptomatic group in comparison to $(21.7 \%)$ in symptomatic group $(\mathrm{p}=0.00)$.

There was a statistically significant difference in interpretation of hyperprolactinemia between the symptomatic and asymptomatic group as patients with free prolactin represented $(86.1 \%)$ of the studied sample in the asymptomatic group and (87.0\%) in the symptomatic group while patients with macroprolactin were $(13.2 \%)$ in the asymptomatic group in comparison to $(2.2 \%)$ in symptomatic group and patients with borderline level were $(0.7 \%)$ in the asymptomatic group in comparison to $(10.9 \%)$ in symptomatic group $(\mathrm{p}=0.00)$. There was a statistically significant difference between total prolactin and post-PEG PRL in which the mean of total PRL was $2413.6 \pm 1486.0$ and the mean of post-PEG PRL was $1759.37 \pm 1260.5(p=0.000)$ which was following the result of Nedjeljka Ruljancic et al 2021 [25]. There was a statistically significant difference in total Prolactin between male and female in which the mean for female (2689.7 \pm 1552.8$)$ were significantly higher than in males $(1410.4 \pm 443.9)(p=0.000)$, Also, there was a statistically significant difference macroprolactin between male and female in which the mean for female $(721.7 \pm 495.3)$ were significantly higher in than in males $(408.9 \pm 290.8)(\mathrm{p}=0.000)$, which was in line with the study of Young-Min Park et al 2016 [26].

There was a statistically significant difference in the type of prolactin between the symptomatic and asymptomatic group as total prolactin mean (2208.3) of the studied sample in the asymptomatic group and (3056.1) in the symptomatic group while patients with corrected prolactin mean were (1589.2) in the asymptomatic group in comparison to (2292) in symptomatic group ( $\mathrm{t}$-test $=0.001$ ). Meanwhile, there was no significant difference in the mean of MaPRL between the asymptomatic and symptomatic groups in the mean of MaPRL ( $\mathrm{t}$-test $=0.007$ ). The treatment of samples with PEG produced a reduction in PRL level in all cases with a mean reduction of $28.3 \%$. There was no significant difference in medications used between the symptomatic and asymptomatic group as risperidone represented $(81.3 \%)$ of the studied sample in the asymptomatic group and (18.7\%) in the symptomatic group while haloperidol represented (64.3\%) in the asymptomatic group in comparison to (35.7\%) in the symptomatic group while Flupenthixol Decanoate represented $(72.2 \%)$ in the asymptomatic group in comparison to $(27.8 \%)$ in symptomatic group and olanzapine represented (88.9\%) in the asymptomatic group in comparison to $(11.1 \%)$ $(p=0.079)$ which was on the contrary of the study of Young-Min Park et al 2016.

The mean of total PRL \& MaPRL was higher in patients who were receiving haloperidol more than the other medications. (Table $1 \&$ Figure 1). In our study there was a positive correlation between total prolactin and macroprolactin levels $(\mathrm{r}=0.59, \mathrm{p}<$ 0.01) (Figure 2).

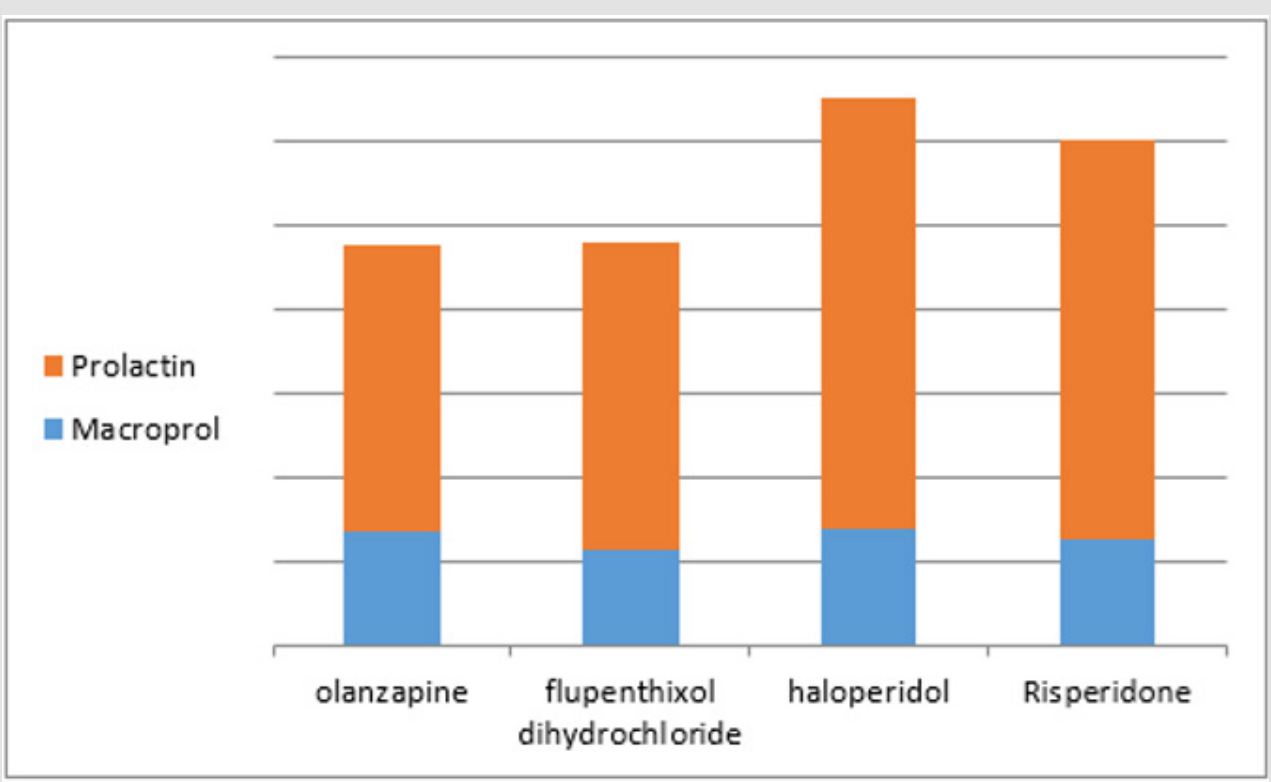

Figure 1: The mean of PRL \& MaPRL in Olanzapine, Flupenthixol Decanoate, and Haloperidol \& Risperidone. (The figure shows that the mean of the total prolactin level and the mean of macroprolactin levels were higher in patients receiving haloperidol that in other medicines). 


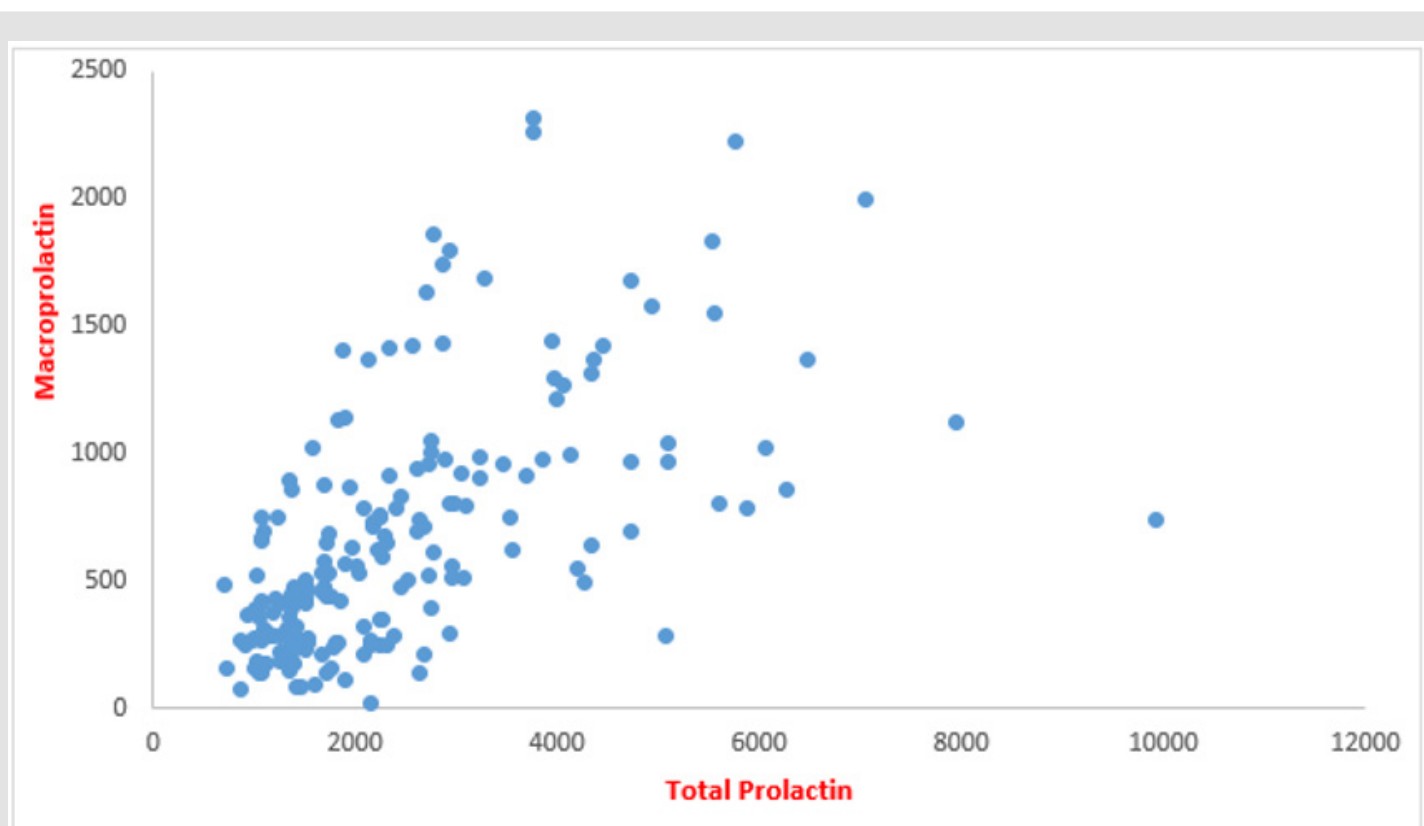

Figure 2: Correlation between total PRL \& MaPRL (The figure shows the positive correlation between the values of the prolactin level and the values of macroprolactin levels).

Table 1: Mean of Total PRL \& MaPRL of Olanzapine, Haloperidol, and Risperidone \& Flupenthixol Decanoate.

\begin{tabular}{|c|c|c|c|c|c|c|c|c|c|c|c|c|}
\hline & \multicolumn{3}{|c|}{ Olanzapine $(n=9)$} & \multicolumn{3}{|c|}{ Haloperidol $(n=56)$} & \multicolumn{3}{|c|}{ Risperidone ( $n=107$ ) } & \multicolumn{3}{|c|}{$\begin{array}{c}\text { Flupenthixol } \\
\text { Decanoate }(n=18)\end{array}$} \\
\hline & YES & $\begin{array}{c}\text { NO } \\
\text { Mean } \pm \\
\text { SD }\end{array}$ & Sig. & YES & $\begin{array}{c}\text { NO } \\
\text { Mean } \pm \\
\text { SD }\end{array}$ & Sig. & YES & NO & Sig. & YES & $\begin{array}{c}\text { NO } \\
\text { Mean } \pm \\
\text { SD }\end{array}$ & Sig. \\
\hline $\begin{array}{l}\text { Total } \\
\text { PRL }\end{array}$ & $\begin{array}{c}\text { Mean } \pm \\
\text { SD } \\
1710.7 \pm \\
893.7\end{array}$ & $\begin{array}{c}2428.4 \pm \\
1482.0\end{array}$ & $\begin{array}{c}\mathrm{t}=- \\
1.438 \\
\mathrm{p}=0.152\end{array}$ & $\begin{array}{c}\text { Mean } \pm \\
\text { SD } \\
2560.9 \\
\pm 1691.1\end{array}$ & $\begin{array}{c}2331.4 \pm \\
1358.9\end{array}$ & $\begin{array}{l}t=1.021 \\
p=0.309\end{array}$ & $\begin{array}{c}\text { Mean } \pm \\
\text { SD } \\
2388.3 \pm \\
1397.5\end{array}$ & $\begin{array}{c}\text { Mean } \pm \\
\text { SD } \\
2446.2 \pm \\
1601\end{array}$ & $\begin{array}{l}t=0.265 \\
p=0.791\end{array}$ & $\begin{array}{c}\text { Mean } \pm \\
\text { SD } \\
1829.4 \pm \\
880.5\end{array}$ & $\begin{array}{c}2478.4 \pm \\
1530.5\end{array}$ & $\begin{array}{l}t=-1.765 \\
p=0.079\end{array}$ \\
\hline MaPRL & $\begin{array}{c}679.7 \pm \\
664.9\end{array}$ & $\begin{array}{c}650.9 \pm \\
467.7\end{array}$ & $\begin{array}{c}\mathrm{t}=- \\
0.177 \\
\mathrm{p}=0.860\end{array}$ & $\begin{array}{l}700.8 \pm \\
467.5\end{array}$ & $\begin{array}{c}628.2 \pm \\
480.6\end{array}$ & $\begin{array}{l}t=-1.007 \\
p=0.315\end{array}$ & $\begin{array}{c}628.2 \pm \\
474.0\end{array}$ & $\begin{array}{l}687.7 \pm \\
479.4\end{array}$ & $\begin{array}{c}\mathrm{t}=- \\
0.853 \\
\mathrm{p}=0.395\end{array}$ & $\begin{array}{c}567.0 \pm \\
316.5\end{array}$ & $\begin{array}{c}665.8 \pm \\
491.7\end{array}$ & $\begin{array}{c}\mathrm{t}=- \\
0.833 \\
\mathrm{p}=0.406\end{array}$ \\
\hline
\end{tabular}

\section{Discussion}

Macroprolactin was described for the first time by Wittaker et al in 1981 when they reported a case with hyperprolactinemia without the common symptoms of amenorrhea, galactorrhea, and infertility. It was found that the high molecular weight MaPRL accounted for the majority of PRL in this patient by using GFC. It is PRL-IgG immunocomplex in which endogenous IgG molecule is directed against epitopes on $\mathrm{N}$ - and C-terminal restudies of monomeric PRL [27]. In the present study, treatment of the sera of patients with high prolactin with $25 \%$ PEG 6000 was done leading to precipitation of macroprolactin and free prolactin level was measured in the supernatant. This method is the easiest and cheapest method for detecting MaPRL but it can precipitate monomeric PRL also with MaPRL leads to a reduction of post-PEG PRL values in all serum samples with a mean reduction of $28.3 \%$ in this study. In our study, we used the most common cut-off $<40 \%$ recovery rate to consider the case positive for macroprolactinemia. It has been reported that the $40 \%$ cutoff is $100 \%$ sensitive to confirm the presence of MaPRL while using a $60 \%$ cutoff does not report significantly more patients with significant macroprolactinemia [28].

In our study, $10.5 \%$ were detected with macroprolactin predominance which is consistent with studies that have been documented in the United States which found that about $10 \%$ of hyperprolactinemic patients have MaPRL as a predominant form of PRL but most of the studies conducted in Europe have shown that the predominance of MaPRL is usually above 20\% [27-29]. $95 \%$ of cases of MaPRL were asymptomatic and $5 \%$ were complaining of amenorrhea. Although MaPRL is biologically inactive the symptoms could be co-incidence or intermittent dissociation of macroprolactin from IgG molecule could happen and causing the symptoms as suggested by Hattori $N$ et al in 1997 [30]. Suliman AM et al also explained that although Big-Big PRL may be the 
predominant molecular form in MaPRL, monomeric PRL may simultaneously be found in excess. Therefore, MaPRL could be associated with monomeric hyperprolactinemia which leads to the development of symptoms of hyperprolactinemia [31]. In our study prolactin levels and macroprolactin levels were significantly higher in females than males as the previous results of Johnsen, et al. [3233]. The antipsychotic-induced hyperprolactinemia is related to differential D2 receptor affinity, 5-hydroxytryptamine receptor affinity, and blood-brain disposition of antipsychotics [34].

Risperidone is one of the anti-psychotics that have poor bloodbrain barrier penetration and high concentrations of risperidone exist in the pituitary [35]. In our study risperidone appeared to increase prolactin in $56.3 \%$ of patients, haloperidol was the second medicine which increased PRL in $29.5 \%$ of patients, Flupenthixol Decanoate increased PRL in $9.5 \%$ of patients and Olanzapine was the fourth medicine which increased PRL in $4.7 \%$ of patients. In our study, there was no significant difference in medications used between the symptomatic and asymptomatic groups. The mean prolactin and macroprolactin levels were higher in patients who were receiving haloperidol than the other medicines but was no statistically significant difference between them. Beda-Maluga et al. reported that most patients with a PRL concentration higher than $100 \mathrm{ng} / \mathrm{ml}$ (2127 uIU/mL) had true hyperprolactinemia, although significant macroprolactinemia might be present in some cases [36]. The American Association of Clinical Endocrinologists recommends using a 25-150 ng/mL (532 - $3191 \mathrm{uIU} / \mathrm{mL}$ ) interval to search for MaPRL, and the Endocrine Society suggests testing every asymptomatic patient for hyperprolactinemia [37]. Our study shed light on the prevalence of macroprolactin in patients receiving antipsychotics by measuring the prolactin level by COBAS e411 analyzer, we suggest in the future study increasing the number of patients and studying the prevalence of macroprolactin using different platforms other than COBAS analyzers.

\section{Conclusion}

Macroprolactin predominance in hyperprolactinemia patients receiving antipsychotics was $10.5 \%$ which is in line with many kinds of literature. Haloperidol was associated with increased total and macroprolactin levels more than other drugs. So, we recommend investigation for macroprolactin predominance in every hyperprolactinemia patient especially the asymptomatic cases to avoid unnecessary radiological imaging and treatment.

\section{Ethical Considerations}

The authors declare that they got approval from the hospital administration and DGHS-Governorate of Muscat to access the electronic files of the patients and collect the medical history and data. The authors declare that no patient data appear in this article. In this research, there was no direct contact with patients, so there was no need for their consent.

\section{Acknowledgment}

The authors would like to thank Dr. Sameh Yousry Bondok for his valuable help and support in this study.

\section{Conflicts of Interest}

The authors declare no conflict of interest.

\section{References}

1. Melmed S, Kleinberg D, Larsen PR, Kronenberg HM, Melmed S, et al. (2003) Williams Textbook of Endocrinology. Philadelphia: Saunders. Anterior pituitary, p. 177-279.

2. ME Freeman, B Kanyicska, A Lerant, G Nagy (2000) Prolactin: Structure, function, and regulation of secretion. Physiological Reviews 80: 15231631.

3. S Shelly, M Boaz, H Orbach (2012) Prolactin and autoimmunity. Autoimmunity Reviews 11(6-7): A465-A470.

4. Leite V, Cosby H, Sobrihno LG, MA Fresnoza, MA Santos, et al. (1992) Characterization of big, big prolactin in patients with hyperprolactinemia. Clin Endcrinol 37: 365-372.

5. Karolina Beda Maluga, Hanna P, Jan K, Marke P, Jacek S, et al. (2011) The detection of macroprolactin by precipitation and ultrafiltration methods. Endokrynol Pol 62(6): 529-536.

6. ME Molitch (1992) Pathologic hyperprolactinemia. Endocrinology and Metabolism Clinics of North America 21(4): 877-901.

7. K Berinder, I Stackenäs, O Akre, AL Hirschberg, AL Hulting (2005) Hyperprolactinaemia in 271 women: Up to three decades of clinical follow-up. Clinical Endocrinology 63(4): 450-455.

8. N Hattori, T Ishihara, K Ikekubo, K Moridera, M Hino, et al. (1992) Autoantibody to human prolactin in patients with idiopathic hyperprolactinemia. Journal of Clinical Endocrinology and Metabolism 75(5): 1226-1229.

9. N Hattori, T Ishihara, Y Saiki, A Shimatsu (2010) Macroprolactinaemia in patients with hyperprolactinaemia: Composition of macroprolactin and stability during long-term follow up. Clinical Endocrinology 73(6): 792-797.

10. Hattori N (2003) Macroprolactinemia: A new cause of hyperprolactinemia. J Pharmacol Sci 92: 171-177.

11. Hattori N, Nakayama $\mathrm{Y}$, Kitagawa $\mathrm{K}$, Tiesong Li, Chiyoko Inagaki, et al. (2007) Development of anti-PRL (prolactin) autoantibodies by homologous PRL in rats: A model for macroprolactinemia. Endocrinology 148: 2465-2470.

12. Leslie H, Courtney CH, Bell PM, DR McCance, PK Ellis, et al. (2001) Laboratory and clinical experience in 55 patients with macroprolactinemia identified by a simple polyethylene glycol precipitation method. J Clin Endocrinol Metab 86: 2743-2746.

13. Vallette Kasic S, Morange Ramos I, Selim A, Ginette Gunz, Sophie Morange, et al. (2002) Macroprolactinemia revisited: A study on 106 patients. J Clin Endocrinol Metab 87: 581-588.

14. Alfonso A, Rieniets KI, Vigersky RA (2006) Incidence and clinical significance of elevated macroprolactin levels in patients with hyperprolactinemia. Endocrinol Pract 12(3): 275-280.

15. Beda K, Winczyk K (2009) Makroprolaktyna - występowanie, metody diagnostyczne i znaczenie kliniczne. FML 36: 87-110.

16. Olukoga AO, Kane JW (1999) Macroprolactinaemia: Validation and application of the polyethylene glycol precipitation test and clinical characterization of the condition. Clinical endocrinology 51(1): 119126. 
17. Smith TP, Kavanagh L, Healy ML, McKenna TJ (2007) Technology insight: Measuring prolactin in clinical samples. Nature clinical practice 3: 279289.

18. Gibney J, Smith TP, McKenna TJ (2005) The impact on clinical practice of routine screening for macroprolactin. The Journal of clinical endocrinology and metabolism 90(7): 3927-3932.

19. Cavaco B, Prazeres S, Santos MA, Sobrinho LG, Leite V (1999) Hyperprolactinemia due to big-big prolactin is differently detected by commercially available immunoassays. J Endocrinol Invest 22(3): 203208.

20. Vallette Kasic S, Morange Ramos I, Selim A, Ginette Gunz, Sophie Morange, et al. (2002) Macroprolactinemia revisited: A study on 106 patients. The Journal of clinical endocrinology and metabolism 87: 581-588.

21. Ana Maia Silva, Paula Martins da Costa, Ana Pacheo, Jose Carlos Oliveria, Claudia Freitas (2014) Assessment of macroprolactinemia by polyethylene glycol precipitation method. Rev Port Endocrinol Diabetes Metab 9(1): 25-28.

22. Pascoe LD, Duran RG, Contreras HI, Manuel AL, Blanco FF, et al. (2001) Frequency of macroprolactinaemia due to antibodies against prolactin in pregnant woman. J Clin Endocrinol Metab 86(2): 924-929.

23. Gilson G, Schmit P, Thix J, Hoffman J, Humbel R (2001) Prolactin results for samples containing macroprolactin are method and sample dependent. Clin Chem 47: 331-333.

24. Schlechte JA (2002) The macroprolactin problem. J Clin Endocrinol Metab 87(12): 5408-5409.

25. Nedjeljka Ruljancic, Ana Bakliza, Sandra Vuk Pisk, Natko Geres, Katarina Matic, et al. (2021) Antipsychotics-induced hyperprolactinemia and screening for macroprolactin. Biochem Med (Zagreb) 31(1): 010707.

26. Young Min Park, Seung hwan Lee, Bun Hee LEE, Kyu Young Lee, Kye Seong Lee, et al. (2016) Prolactin and macroprolactin levels in psychiatric patients receiving antipsychotics: A preliminary study. Psychiatry Research 238: 184-189.

27. Wittaker PG, Wilcox T, Lind T (1981) Maintained fertility in a patient with hyperprolactinemia due to big - big prolactin. J Clin Endocrinol Metab 53(4): 863-866

28. Thomas P Smith, Abdulwahab M Suliman, Michael N Fahie Wilson, T Joseph McKenna (2002) Gross Variability in the Detection of Prolactin in Sera Containing Big Big Prolactin (Macroprolactin) by Commercial Immunoassays. The Journal of Clinical Endocrinology \& Metabolism 87(12): 5410-5415.

29. Sapin R, Kertesz G (2003) Macroprolactin detection by precipitation with Protein a-Sepharose: A rapid screening method compared with polyethylene glycol precipitation. Clin Chem 49(3): 502-504.

30. Hattori N, Inagaki C (1997) Anti-prolactin (PRL) autoantibodies cause asymptomatic hyperprolactinemia: Bioassay and clearance studies pf PRL-immunoglobulin G complex. J Clin Endocrinol Metabl 82(9): 31073110.

31. Suliman AM, Smith TP, Gibney J, McKenna TJ (2003) Frequent misdiagnosis and mismanagement of hyperprolactinemic patients before the introduction of macroprolactin screening: Application of a new strict laboratory definition of macroprolactinemia. Clinical Chemistry 49(9): 1504-1509.

32. Kinon BJ, Gilmore JA, Liu H, Halbreich UM (2003) Hyperprolactinemia in response to antipsychotic drugs: Characterization across comparative clinical trials. Psychoneuroendocrinology 28(2): S69-S82.

33. Johnsen E, Kroken RA, Abaza M, Olberg H, Jorgensen HA (2008) Antipsychotic induced hyperprolactinemia: A cross-sectional survey. J Clin Psycho pharmacol 28(6): 686-690.

34. Fitzgerald P, Dinan TG (2008) Prolactin and dopamine: What is the connection? A review article. J Psycho pharmacol 22(2): S12-S19.

35. Kapur S, Langlois X, Vinken P, Megens AA, DeCoster R, et al. (2002) The differential effects of atypical antipsychotics on prolactin elevation are explained by their differential blood-brain disposition: A pharmacological analysis in rats. J Pharmacol Exp Ther 302(3): 11291134

36. Beda Maluga K, Pisarek H, Komorowski J, Swietoslawski J, Fuss Chmielewska J, et al. (2014) Evaluation of hyperprolactinaemia with the use of the intervals for prolactin after macroforms separation. J Physiol Pharmacol 65(3): 359-364.

37. Samson SL, Hamrahian AH, Ezzat S (2015) American Association of Clinical Endocrinologists. American College of Endocrinology disease state clinical review: Clinical relevance of macroprolactin in the absence or presence of true hyperprolactinemia. Endocr Pract 21(12): 14271435.
ISSN: 2574-1241

DOI: $10.26717 /$ BJSTR.2021.37.006015

Loai Ali Al Mortada Al Wasify. Biomed J Sci \& Tech Res

cC) (9) This work is licensed under Creative Commons Attribution 4.0 License

Submission Link: https://biomedres.us/submit-manuscript.php

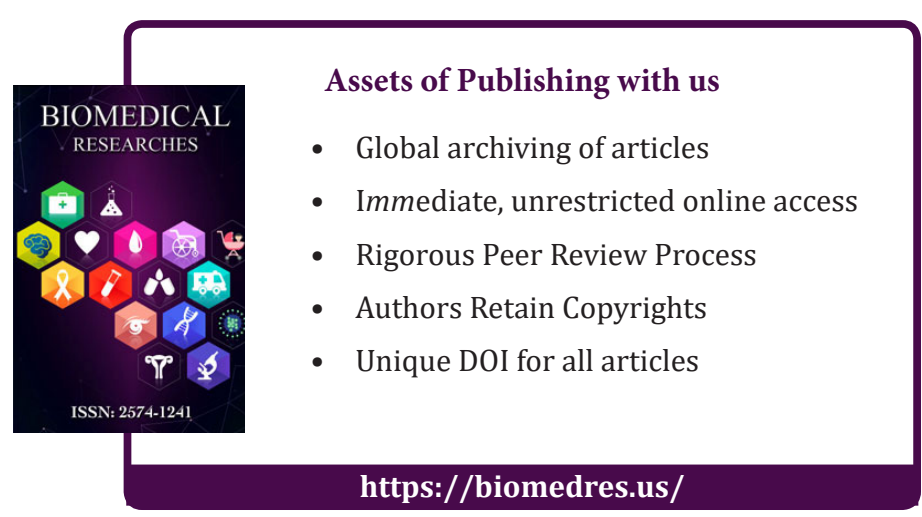

